\title{
The Quaternary Interpolating Scheme for Geometric Design
}

\author{
Shahid S. Siddiqi ${ }^{1}$ and Muhammad Younis ${ }^{2}$ \\ ${ }^{1}$ Department of Mathematics, University of the Punjab, Lahore 54590, Pakistan \\ ${ }^{2}$ Centre for Undergraduate Studies, University of the Punjab, Lahore 54590, Pakistan
}

Correspondence should be addressed to Muhammad Younis; younis.pu@gmail.com

Received 18 February 2013; Accepted 27 March 2013

Academic Editors: A. Castelo, C. Cusano, F. Giannini, C. Joslin, G. Patanè, and C.-M. Wang

Copyright (C) 2013 S. S. Siddiqi and M. Younis. This is an open access article distributed under the Creative Commons Attribution License, which permits unrestricted use, distribution, and reproduction in any medium, provided the original work is properly cited.

A novel 4-point interpolating subdivision scheme is presented, which generates the family of $C^{2}$ limiting curves and its limiting function has support on $[-7 / 3,7 / 3]$. It behaves better than classical 4-point binary and ternary schemes with the same approximation order in many aspects that it has smaller support size, higher smoothness, and is computationally more efficient. The proposed nonstationary scheme can reproduce the functions of linear spaces spanned by $\{1, \sin (\alpha x), \cos (\alpha x)\}$ for $0<\alpha<\pi / 3$. Moreover, some examples are illustrated to show that the proposed scheme can also reproduce asteroids, cardioids, and conic sections as well.

\section{Introduction}

Subdivision schemes have become one of the most important, significant, and emerging modelling tools in computer applications, medical image processing, scientific visualization, reverse engineering, computer aided geometric designing, and so forth, because of their simple, elegant, and efficient ways to create smooth curves from initial control polygon by subdividing them according to some refining rules, recursively. These refining rules take the initial polygon to produce a sequence of finer polygons converging to a smooth limiting curve.

In the field of nonstationary subdivision schemes, binary and ternary schemes are present in the literature [1-6]. But the quaternary schemes are not discussed as others. The aim of this paper is to introduce a quaternary nonstationary scheme which reproduces the linear functions spanned by $\{1, \sin (\alpha x), \cos (\alpha x)\}$. Moreover, the proposed scheme can also generate asteroids, cardioids, and in particular conic sections like circles, ellipses, and so forth. Some properties of the proposed scheme (like support size and symmetry of basic limit function, approximation order, convergence, and smoothness) have also been investigated. Besides the convergence and approximation order, the two important and interesting properties of basic limit function of a subdivision scheme are the support size and the smoothness. They are commonly manipulated with each other in the sense that a higher degree of smoothness generally requires a larger support, thus leading to a more global influence of each initial data value on the limit function. Raising the arity of the subdivision scheme provides a way to overcome this problem to some extent. The proposed scheme has smaller support and higher smoothness than the classical binary 4-point scheme, and all other schemes reproduce cubic polynomials by construction whether they are stationary or nonstationary $[1-4,6,7]$.

As interpolating subdivision schemes retain the points of stage $k$ as the subset of the points of next stage $k+1$, thus the structure of a quaternary 4-point interpolating scheme in general form can be written as

$$
\begin{aligned}
& f_{4 i}^{k+1}=f_{i}^{k} \\
& f_{4 i+1}^{k+1}=a_{0}^{(k)} f_{i-1}^{k}+a_{1}^{(k)} f_{i}^{k}+a_{2}^{(k)} f_{i+1}^{k}+a_{3}^{(k)} f_{i+2}^{k} \\
& f_{4 i+2}^{k+1}=b_{0}^{(k)} f_{i-1}^{k}+b_{1}^{(k)} f_{i}^{k}+b_{2}^{(k)} f_{i+1}^{k}+b_{3}^{(k)} f_{i+2}^{k} \\
& f_{4 i+3}^{k+1}=a_{3}^{(k)} f_{i-1}^{k}+a_{2}^{(k)} f_{i}^{k}+a_{1}^{(k)} f_{i+1}^{k}+a_{0}^{(k)} f_{i+2}^{k},
\end{aligned}
$$

where the coefficients $\left\{a_{j}^{(k)}\right\}_{j=0,1,2,3}$ and $\left\{b_{j}^{(k)}\right\}_{j=0,1,2,3}$ are chosen to satisfy the relations $\sum_{j=0}^{3} a_{j}^{(k)}=1$ and $\sum_{j=0}^{3} b_{j}^{(k)}=1$, respectively, for a large value of $k$. 
This paper is organized as follows. In Section 2 the basic notion and definitions of the quaternary subdivision scheme are considered. The construction of the proposed scheme has been shown in Section 3. In Section 4, the convergence and smoothness $C^{2}$ of the scheme are being calculated using the theory of asymptotic equivalence. The basic limit function and its properties are being discussed in Section 5. The conclusion is drawn in Section 6.

\section{Preliminaries}

A quaternary subdivision scheme, to generate smooth curves or surfaces, is defined as the limit of refined control polygons or meshes, according to some refinement rules, recursively, in which the set of control points $f^{k}=\left\{f_{i}^{k} \in R \mid i \in Z\right\}$ of polygon at $k$ th level is mapped to a refined polygon to generate the new set of control points $f^{k+1}=\left\{f_{i}^{k+1} \in R \mid i \in\right.$ $Z\}$ at the $(k+1)$ st level by applying the following subdivision rule:

$$
f_{i}^{k+1}=\left\{S_{a^{(k)}} f^{k}\right\}_{i}=\sum_{j \in Z} a_{i-4 j}^{(k)} f_{j}^{k} \quad \forall i \in Z
$$

with the sequence of finite sets of real coefficients $\left\{a_{i}^{(k)}: k \geq\right.$ $0\}$, constitute the so-called mask of the subdivision scheme. If the mask of a scheme is independent of $k$ (namely, if $a^{(k)}=a$ for all $k \geq 0$ ), then it is called stationary $S_{a}$; otherwise it is called nonstationary $S_{a^{(k)}}$.

The convergent subdivision scheme $S_{a}$ with the corresponding mask $\left\{a_{i}, i \in Z\right\}$ necessarily satisfies

$$
\sum_{j \in Z} a_{4 j+l}=1, \quad l=0,1,2,3 .
$$

It follows that the symbol of a convergent subdivision scheme satisfies the conditions

$$
a(1)=4, \quad a\left(e^{((2 i \pi / 4) j)}\right)=0, \quad j=1,2,3 .
$$

For this, we introduce a symbol called the Laurent polynomial

$$
a(z)=\sum_{i \in Z} a_{i} z^{i}
$$

of a mask $\left\{a_{i}, i \in Z\right\}$ with finite support. In view of Dyn [10], the sufficient and necessary conditions for a uniform convergent scheme are defined as follows.

A quaternary subdivision scheme $S_{a}$ is uniformly convergent if and only if there is an integer $L \geq 1$, such that

$$
\left\|\left(\frac{1}{4} S_{a_{1}}\right)^{L}\right\|_{\infty}<1
$$

and subdivision $S_{a_{1}}$ with symbol $a_{1}(z)$ is related to $S_{a}$ with symbol $a(z)$, where $a_{1}(z)=\left(4 z^{3} /\left(1+z+z^{2}+z^{3}\right)\right) a(z)$ satisfying the property

$$
d f^{k}=S_{1} d f^{k-1}, \quad k=1,2, \ldots,
$$

where $f^{k}=S_{a}^{k} f^{0}=\left\{f_{i}^{k}: i \in Z\right\}$ and $d f^{k}=\left\{\left(d f^{k}\right)_{i}=4^{k}\left(f_{i+1}^{k}-\right.\right.$ $\left.\left.f_{i}^{k}\right): i \in Z\right\}$. The norm $\left\|S_{a}\right\|_{\infty}$ of a subdivision scheme $S_{a}$ with a mask $a_{i}, \quad i \in Z$, is defined by $\left\|S_{a}\right\|_{\infty}$ and

$$
\left\|\left(\frac{1}{4} S_{a}\right)^{L}\right\|_{\infty}=\max \left\{\sum_{\beta}\left|a_{\gamma+4^{L} \beta}^{[L]}\right|: \gamma=0,1, \ldots, 4^{L}-1\right\},
$$

where

$$
a_{m}^{[L]}(z)=\prod_{j=0}^{L-1} a_{m}\left(z^{4^{j}}\right) .
$$

The notation of convergence for quaternary subdivision $C^{m}$ scheme, similar to that given by Dyn and Levin in [11] for a binary scheme, is given in the following.

Definition 1. A quaternary subdivision scheme $\left\{S_{a^{k}}\right\}$ is said to be convergent if for every initial data $f^{0} \in l^{\infty}$ there exists a limit function $f \in C^{m}(R)$ such that

$$
\lim _{k \rightarrow \infty} \sup _{i \in Z}\left|f_{i}^{k}-f\left(4^{-k} i\right)\right|=0
$$

and $f$ is not identically zero for some initial data $f^{0}$.

Definition 2. Two quaternary subdivision schemes $\left\{S_{a^{(k)}}\right\}$ and $\left\{S_{b^{(k)}}\right\}$ are said to be asymptotically equivalent if

$$
\sum_{k=1}^{\infty}\left\|S_{a^{(k)}}-S_{b^{(k)}}\right\|_{\infty}<\infty,
$$

where

$$
\begin{aligned}
& \left\|S_{a^{(k)}}\right\|_{\infty} \\
& \quad=\max \left\{\sum_{j=Z}\left|a_{4 i}^{(k)}\right|, \sum_{i \in Z}\left|a_{4 i+1}^{(k)}\right|, \sum_{i \in Z}\left|a_{4 i+2}^{(k)}\right|, \sum_{i \in Z}\left|a_{4 i+3}^{(k)}\right|\right\} .
\end{aligned}
$$

Theorem 3. Let $\left\{S_{a^{k}}\right\}$ and $\left\{S_{a}\right\}$ be quaternary nonstationary and stationary subdivision schemes, respectively, which are said to be asymptotically equivalent if they have finite masks of the same support. If stationary scheme $\left\{S_{a}\right\}$ is $C^{m}$ and

$$
\sum_{k=0}^{\infty} 4^{m k}\left\|S_{a^{(k)}}-S_{a}\right\|_{\infty}<\infty
$$

then nonstationary scheme $\left\{S_{a^{k}}\right\}$ is also $C^{m}$.

\section{The Nonstationary Subdivision Scheme}

In this paper, an interpolating 4-point nonstationary scheme is presented which is associated with Lagrange-like cubic trigonometric interpolants. For this, consider a space $\tau:=\operatorname{span}\{1, \sin (\alpha x), \cos (\alpha x)\}$ for some $\alpha$ such that $0<\alpha<\pi$, for the data set, $D=\left\{\left(x_{-1}, f\left(x_{-1}\right)\right)\right.$, $\left.\left(x_{0}, f\left(x_{0}\right)\right),\left(x_{1}, f\left(x_{1}\right)\right),\left(x_{2}, f\left(x_{2}\right)\right)\right\}$, and also consider the function,

$$
L(x)=\sum_{j=-1}^{2} f\left(x_{j}\right) L_{j}(x)
$$


where

$$
L_{j}(x)=\prod_{k=-1, k \neq j}^{2} \frac{\sin \left((\alpha / 4)\left(x-x_{k}\right)\right)}{\sin \left((\alpha / 4)\left(x_{j}-x_{k}\right)\right)},
$$

is a unique function in $\tau$ which interpolates the data set with having minimum amplitude among other interpolants from $\tau$ [12], where the function $L(x)$ is called the trigonometric Lagrange-like interpolant of the previous data. Since the function $L_{j}(x)$ is defined for $x_{j}=j, j=-1,0,1,2$, where $L_{j}(x)$ is represented by $L_{j}^{D^{m}}(x), m=1,2,3$ for the following discussion.

(i) If $D^{1}=\left\{x_{j}=j: j=-1,0,1,2\right.$ and $\left.x^{\prime}=1 / 4\right\}$, then

$$
\begin{aligned}
& L_{-1}^{D^{1}}\left(x^{\prime}\right)=-\frac{\sin (\alpha / 16) \sin (3 \alpha / 16) \sin (7 \alpha / 16)}{\sin (\alpha / 4) \sin (2 \alpha / 4) \sin (3 \alpha / 4)}, \\
& L_{0}^{D^{1}}\left(x^{\prime}\right)=\frac{\sin (3 \alpha / 16) \sin (5 \alpha / 16) \sin (7 \alpha / 16)}{\sin ^{2}(\alpha / 4) \sin (2 \alpha / 4)} \\
& L_{1}^{D^{1}}\left(x^{\prime}\right)=\frac{\sin (\alpha / 16) \sin (5 \alpha / 16) \sin (7 \alpha / 16)}{\sin ^{2}(\alpha / 4) \sin (2 \alpha / 4)} \\
& L_{2}^{D^{1}}\left(x^{\prime}\right)=-\frac{\sin (\alpha / 16) \sin (3 \alpha / 16) \sin (5 \alpha / 16)}{\sin (\alpha / 4) \sin (2 \alpha / 4) \sin (3 \alpha / 4)} .
\end{aligned}
$$

(ii) If $D^{2}=\left\{x_{j}=j: j=-1,0,1,2\right.$ and $\left.x^{\prime \prime}=1 / 2\right\}$, then

$L_{-1}^{D^{2}}\left(x^{\prime \prime}\right)=L_{2}^{D^{2}}\left(x^{\prime \prime}\right)=-\frac{\sin ^{2}(\alpha / 8) \sin (3 \alpha / 8)}{\sin (\alpha / 4) \sin (2 \alpha / 4) \sin (3 \alpha / 4)}$,

$L_{0}^{D^{2}}\left(x^{\prime \prime}\right)=L_{1}^{D^{2}}\left(x^{\prime \prime}\right)=\frac{\sin (\alpha / 8) \sin ^{2}(3 \alpha / 8)}{\sin ^{2}(\alpha / 4) \sin (2 \alpha / 4)}$,

and similarly, it can be obtained for the following.

(iii) If $D^{3}=\left\{x_{j}=j: j=-1,0,1,2\right.$ and $\left.x^{\prime \prime \prime}=3 / 4\right\}$, then

$L_{-1}^{D^{1}}\left(x^{\prime}\right)=L_{2}^{D^{3}}\left(x^{\prime \prime \prime}\right), \quad L_{0}^{D^{1}}\left(x^{\prime}\right)=L_{1}^{D^{3}}\left(x^{\prime \prime \prime}\right)$,

$L_{1}^{D^{1}}\left(x^{\prime}\right)=L_{0}^{D^{3}}\left(x^{\prime \prime \prime}\right), \quad L_{2}^{D^{1}}\left(x^{\prime}\right)=L_{-1}^{D^{3}}\left(x^{\prime \prime \prime}\right)$.
After that, adjust the previous values to the masks $\left\{a_{j}^{(k)}\right\}_{j=0,1,2,3}$ of the subdivision scheme (1) in the following manner:

$$
\begin{aligned}
a_{0}^{(k)} & =\frac{\sin \left(\alpha / 2^{k+4}\right) \sin \left(3 \alpha / 2^{k+4}\right) \sin \left(7 \alpha / 2^{k+4}\right)}{\sin \left(\alpha / 2^{k+2}\right) \sin \left(2 \alpha / 2^{k+2}\right) \sin \left(3 \alpha / 2^{k+2}\right)} \\
& =L_{-1}^{D^{1}}\left(x^{\prime}\right)=L_{2}^{D^{3}}\left(x^{\prime \prime \prime}\right) \\
a_{1}^{(k)} & =\frac{\sin \left(3 \alpha / 2^{k+4}\right) \sin \left(5 \alpha / 2^{k+4}\right) \sin \left(7 \alpha / 2^{k+4}\right)}{\sin ^{2}\left(\alpha / 2^{k+2}\right) \sin \left(2 \alpha / 2^{k+2}\right)} \\
& =L_{0}^{D^{1}}\left(x^{\prime}\right)=L_{1}^{D^{3}}\left(x^{\prime \prime \prime}\right) \\
a_{2}^{(k)} & =\frac{\sin \left(\alpha / 2^{k+4}\right) \sin \left(5 \alpha / 2^{k+4}\right) \sin \left(7 \alpha / 2^{k+4}\right)}{\sin ^{2}\left(\alpha / 2^{k+2}\right) \sin \left(2 \alpha / 2^{k+2}\right)} \\
& =L_{1}^{D^{1}}\left(x^{\prime}\right)=L_{0}^{D^{3}}\left(x^{\prime \prime \prime}\right) \\
a_{3}^{(k)} & =\frac{\sin \left(\alpha / 2^{k+4}\right) \sin \left(3 \alpha / 2^{k+4}\right) \sin \left(5 \alpha / 2^{k+4}\right)}{\sin \left(\alpha / 2^{k+2}\right) \sin \left(2 \alpha / 2^{k+2}\right) \sin \left(3 \alpha / 2^{k+2}\right)} \\
& =L_{2}^{D^{1}}\left(x^{\prime}\right)=L_{-1}^{D^{3}}\left(x^{\prime \prime \prime}\right),
\end{aligned}
$$

and also we have

$$
\begin{aligned}
& b_{0}^{(k)}=b_{3}^{(k)}=\frac{\sin ^{2}\left(\alpha / 2^{k+3}\right) \sin \left(3 \alpha / 2^{k+3}\right)}{\sin \left(\alpha / 2^{k+2}\right) \sin \left(2 \alpha / 2^{k+2}\right) \sin \left(3 \alpha / 2^{k+2}\right)}, \\
& b_{1}^{(k)}=b_{2}^{(k)}=\frac{\sin \left(\alpha / 2^{k+3}\right) \sin ^{2}\left(3 \alpha / 2^{k+3}\right)}{\sin ^{2}\left(\alpha / 2^{k+2}\right) \sin \left(2 \alpha / 2^{k+2}\right)} .
\end{aligned}
$$

Thus, an interpolating 4-point nonstationary subdivision rule to refine the control polygon is defined in (21), where the mask of proposed scheme satisfies the affine properties, presented by the set of (19) and (20). So, we have

$$
\begin{aligned}
& f_{4 i}^{k+1}=f_{i}^{k} \\
& f_{4 i+1}^{k+1}=-a_{0}^{(k)} f_{i-1}^{k}+a_{1}^{(k)} f_{i}^{k}+a_{2}^{(k)} f_{i+1}^{k}-a_{3}^{(k)} f_{i+2}^{k} \\
& f_{4 i+1}^{k+1}=-b_{0}^{(k)} f_{i-1}^{k}+b_{1}^{(k)} f_{i}^{k}+b_{2}^{(k)} f_{i+1}^{k}-b_{3}^{(k)} f_{i+2}^{k} \\
& f_{4 i+2}^{k+1}=-a_{3}^{(k)} f_{i-i}^{k}+a_{2}^{(k)} f_{i}^{k}+a_{1}^{(k)} f_{i+1}^{k}-a_{0}^{(k)} f_{i+2}^{k} .
\end{aligned}
$$

It may be noted that the points $\left((4 i+1) / 4^{k+1}, f_{4 i+1}^{k+1}\right)$, $\left((4 i+2) / 4^{k+1}, f_{4 i+1}^{k+1}\right)$, and $\left((4 i+3) / 4^{k+1}, f_{4 i+1}^{k+1}\right)$ lie on the Lagrange-like interpolant of the set of points $\{((i-$ 1) $\left./ 4^{k}, f_{i-1}^{k}\right),\left(i / 4^{k}, f_{i}^{k}\right),\left((i+1) / 4^{k}, f_{i+1}^{k}\right)$, and $\left.\left((i+2) / 4^{k}, f_{i+2}^{k}\right)\right\}$. Moreover, the mask of scheme (21) is bounded by the following coefficients:

$$
\begin{aligned}
& a_{0}^{k} \longrightarrow \frac{7}{128}, \quad a_{1}^{k} \longrightarrow \frac{105}{128}, \\
& a_{2}^{k} \longrightarrow \frac{35}{128}, \quad a_{3}^{k} \longrightarrow \frac{5}{128}, \\
& b_{0}^{k}=b_{3}^{k} \longrightarrow \frac{1}{16}, \quad b_{1}^{k}=b_{2}^{k} \longrightarrow \frac{9}{16} .
\end{aligned}
$$


The proof of the previous boundedness can be followed from Lemma 4.

\section{Convergence and Smoothness}

The theory of asymptotic equivalence is used to investigate the convergence and smoothness of the scheme following [11]. For this, some estimations of $a_{i}^{(k)}, i=0,1,2,3$ and $b_{i}^{(k)}, i=$ $0,1,2,3$ used are given in the following lemmas. To prove the lemmas, the following three inequalities are used:

$$
\begin{gathered}
\frac{\sin a}{\sin b} \geq \frac{a}{b} \quad \text { for } 0<a<b<\frac{\pi}{2}, \\
\theta \csc \theta \leq t \csc t \quad \text { for } 0<\theta<t<\frac{\pi}{2}, \\
\cos x \leq \frac{\sin x}{x} \quad \text { for } 0<x<\frac{\pi}{2}
\end{gathered}
$$

Lemma 4. For $k \geq 0$ and $0<\alpha<\pi / 2$, one has

$$
\begin{aligned}
& \text { (i) } \frac{7}{128} \leq a_{0}^{(k)} \leq \frac{7}{128} \frac{1}{\cos ^{3}\left(3 \alpha / 2^{k+1}\right)} \\
& \text { (ii) } \frac{105}{128} \leq a_{1}^{(k)} \leq \frac{105}{128} \frac{1}{\cos ^{3}\left(\alpha / 2^{k+1}\right)} \\
& \text { (iii) } \frac{35}{128} \leq a_{2}^{(k)} \leq \frac{35}{128} \frac{1}{\cos ^{3}\left(\alpha / 2^{k+1}\right)} \\
& \text { (iv) } \frac{5}{128} \leq a_{3}^{(k)} \leq \frac{5}{128} \frac{1}{\cos ^{3}\left(3 \alpha / 2^{k+1}\right)} \\
& \text { (v) } \frac{1}{16} \leq b_{0}^{(k)} \leq \frac{1}{16} \frac{1}{\cos ^{3}\left(\alpha / 2^{k+1}\right)} \\
& \text { (vi) } \frac{9}{16} \leq b_{1}^{(k)} \leq \frac{9}{16} \frac{1}{\cos ^{3}\left(3 \alpha / 2^{k+1}\right)}
\end{aligned}
$$

Proof. To prove the inequality (i)

$$
\begin{aligned}
a_{0}^{(k)} & =\frac{\sin \left(\alpha / 2^{k+4}\right) \sin \left(3 \alpha / 2^{k+4}\right) \sin \left(7 \alpha / 2^{k+4}\right)}{\sin \left(\alpha / 2^{k+2}\right) \sin \left(2 \alpha / 2^{k+2}\right) \sin \left(3 \alpha / 2^{k+2}\right)} \\
& \geq \frac{\left(\alpha / 2^{k+4}\right)\left(3 \alpha / 23^{k+4}\right)\left(7 \alpha / 2^{k+4}\right)}{\left(\alpha / 23^{k+2}\right)\left(2 \alpha / 2^{k+2}\right)\left(3 \alpha / 2^{k+2}\right)}=\frac{7}{128} \\
a_{0}^{(k)} & \leq \frac{21 \alpha^{3}}{2^{3 k+12}} \csc \left(\frac{\alpha}{2^{k+2}}\right) \csc \left(\frac{2 \alpha}{2^{k+2}}\right) \csc \left(\frac{3 \alpha}{2^{k+2}}\right) \\
& \leq \frac{21 \alpha^{3}}{2^{3 k+12}} 36 \csc ^{3}\left(\frac{3 \alpha}{2^{k+1}}\right) \\
& \leq \frac{21 \alpha^{3}}{2^{3 k+12}} 36 \frac{\left(3 \alpha / 2^{k+1}\right)^{3} \cos ^{3}\left(3 \alpha / 2^{k+1}\right)}{\left(\frac{1}{128} \frac{1}{\cos ^{3}\left(3 \alpha / 2^{k+1}\right)} .\right.}
\end{aligned}
$$

The proof of (ii), (iii), (iv), (v), and (vi) can be obtained similarly.
Lemma 5. For some constants $C_{0}, C_{1}, C_{2}, C_{3}, C_{4}$, and $C_{5}$ independent of $k$, one has the following:

$$
\begin{aligned}
& \text { (i) }\left|a_{0}^{(k)}-\frac{7}{128}\right| \leq C_{0} \frac{1}{4^{k}}, \\
& \text { (ii) }\left|a_{1}^{(k)}-\frac{105}{128}\right| \leq C_{1} \frac{1}{4^{k}}, \\
& \text { (iii) }\left|a_{2}^{(k)}-\frac{35}{128}\right| \leq C_{2} \frac{1}{4^{k}}, \\
& \text { (iv) }\left|a_{3}^{(k)}-\frac{5}{128}\right| \leq C_{3} \frac{1}{4^{k}}, \\
& \text { (v) }\left|b_{0}^{(k)}-\frac{1}{16}\right| \leq C_{4} \frac{1}{4^{k}}, \\
& \text { (vi) }\left|b_{1}^{(k)}-\frac{9}{16}\right| \leq C_{5} \frac{1}{4^{k}} .
\end{aligned}
$$

Proof. Inequality (i) can be proved using Lemma 4 So

$$
\text { (i) } \begin{aligned}
\left|a_{0}^{(k)}-\frac{7}{128}\right| & \leq \frac{7}{128}\left(\frac{1-\cos ^{3}\left(3 \alpha /\left(2^{k}+1\right)\right)}{\cos ^{3} \alpha}\right) \\
& \leq \frac{7}{128}\left(\frac{3 \sin ^{2}\left(3 \alpha /\left(2^{k}+1\right)\right)}{2 \cos ^{3} \alpha}\right) \\
& \leq \frac{189 \alpha^{2}}{1024 \cos ^{3} \alpha} \frac{1}{4^{k}} .
\end{aligned}
$$

The proof of (ii), (iii), (iv), (v), and (vi) can be obtained similarly.

Lemma 6. The Laurent polynomial $a^{(k)}(z)$ of the scheme $\left\{S_{a^{(k)}}\right\}$ at the kth level can be written as $a^{(k)}(z)=\left(\left(1+z+z^{2}+\right.\right.$ $\left.\left.z^{3}\right) / 4\right) c^{(k)}(z)$, where

$$
\begin{aligned}
c^{(k)}(z)=4\{ & -a_{3}^{(k)} z^{4}+\left(a_{3}^{(k)}-b_{0}^{(k)}\right) z^{3}+\left(b_{0}^{(k)}-a_{0}^{(k)}\right) z^{2} \\
& +a_{0}^{(k)} z+\left(a_{2}^{(k)}-a_{3}^{(k)}\right) \\
& +\left(b_{1}^{(k)}-b_{0}^{(k)}-a_{2}^{(k)}+a_{3}^{(k)}\right) z^{-1} \\
& +\left(a_{1}^{(k)}-a_{0}^{(k)}-b_{1}^{(k)}+b_{0}^{(k)}\right) z^{-2}+\left(a_{2}^{(k)}-a_{3}^{(k)}\right) z^{-3} \\
& +a_{0}^{(k)} z^{-4}+\left(b_{0}^{(k)}-a_{0}^{(k)}\right) z^{-5}+\left(a_{3}^{(k)}-b_{0}^{(k)}\right) z^{-6} \\
& \left.-a_{3}^{(k)} z^{-7}\right\} .
\end{aligned}
$$

Proof. Since

$$
\begin{aligned}
a^{k(k)}(z)= & -a_{3}^{(k)} z^{7}-b_{3}^{(k)} z^{6}-a_{0}^{(k)} z^{5}+a_{2}^{(k)} z^{3}+b_{2}^{(k)} z^{2} \\
& +a_{1}^{(k)} z+1+a_{1}^{(k)} z^{-1}+b_{1}^{(k)} z^{-2}+a_{2}^{(k)} z^{-3} \\
& -a_{0}^{(k)} z^{-5}-b_{0}^{(k)} z^{-6}-a_{3}^{(k)} z^{-7}
\end{aligned}
$$

therefore using $-a_{0}^{(k)}+a_{1}^{(k)}+a_{2}^{(k)}-a_{3}^{(k)}=1$ and $-b_{0}^{(k)}+b_{1}^{(k)}=1 / 2$ following result can be established $a^{(k)}(z)=\left(\left(1+z+z^{2}+\right.\right.$ $\left.\left.z^{3}\right) / 4\right) b^{(k)}(z)$. 
Lemma 7. The Laurent polynomial $a(z)$ of the scheme $\left\{S_{a}\right\}$ can be written as $a(z)=\left(\left(1+z+z^{2}+z^{3}\right) / 4\right) c(z)$, where

$$
\begin{aligned}
c(z)=\frac{1}{32}\{ & -5 z^{4}-3 z^{3}+z^{2}+7 z+30+34 z^{-1} \\
& \left.+34 z^{-2}+30 z^{-3}+7 z^{-4}+z^{-5}-3 z^{-6}-5 z^{-7}\right\},
\end{aligned}
$$

and the subdivision scheme $\left\{S_{c}\right\}$ corresponding to the symbol $c(z)$ is $C^{1}$.

Proof. The mask defined by the scheme $\left\{S_{c}\right\}$ converges and has the smoothness $C^{1}$ (it can be proved using Laurent polynomial method). If $\left\{S_{c}\right\}$ is $C^{1}$, then $\left\{S_{a}\right\}$ with the mask

$$
\frac{1}{128}[-5,8,-7,0,35,72,105,128,105,72,35,0,-7,8,-5]
$$

is $C^{2}$.

Theorem 8. The scheme defined by (21) converges and has smoothness $C^{2}$.

Proof. To prove the proposed scheme to be $C^{2}$, it is sufficient to show that the scheme corresponding to $c^{k}(z)$ is $C^{1}$ (Theorem (8) given by Dyn and Levin [13]). Since $\left\{S_{c}\right\}$ is $C^{1}$ by Lemma 7, then following Theorem 3, it has been shown that the schemes $\left\{S_{c}^{(k)}\right\}$ and $\left\{S_{c}\right\}$ are asymptotically equivalent. So we have to show that

$$
\sum_{k=0}^{\infty} 4^{k}\left\|S_{c^{(k)}}-S_{c}\right\|_{\infty}<\infty
$$

where

$$
\left\|S_{c^{(k)}}-S_{c}\right\|_{\infty}=\max \left\{\sum_{j=Z}^{\infty}\left|c_{i+4 j}^{(k)}-c_{i+4 j}\right|: i=0,1,2,3\right\} .
$$

From Lemmas 6 and 7, it can be written as

$$
\begin{aligned}
\sum_{j \in Z}^{\infty}\left|c_{4 j}^{(k)}-c_{4 j}\right| & =\left|4 a_{3}^{k}-\frac{5}{32}\right|+\left|4 a_{2}^{k}-4 a_{3}^{k}-\frac{30}{32}\right|+\left|4 a_{0}^{k}-\frac{7}{32}\right| \\
& =4\left|a_{0}^{k}-\frac{7}{128}\right|+4\left|a_{2}^{k}-\frac{35}{128}\right|+8\left|a_{3}^{k}-\frac{5}{128}\right| \\
& =\sum_{j \in Z}^{\infty}\left|c_{4 j+3}^{k}-c_{4 j+3}\right| .
\end{aligned}
$$

TABLE 1: Comparison of the 4-point interpolating subdivision schemes.

\begin{tabular}{lcccc}
\hline Scheme & Type & Continuity & $\begin{array}{c}\text { Approximation } \\
\text { order }\end{array}$ & Support \\
\hline $\begin{array}{l}\text { Binary [8] } \\
\begin{array}{l}\text { Ternary } \\
{[9]}\end{array}\end{array}$ & Stationary & $C^{1}$ & 4 & {$[-3,3]$} \\
$\begin{array}{l}\text { Proposed } \\
(32)\end{array}$ & Stationary & $C^{2}$ & 3 & {$[-2.5,2.5]$} \\
\hline $\begin{array}{l}\text { Binary [1] } \\
\text { Binary [2] }\end{array}$ & Nonstationary & $C^{2}$ & 4 & {$[-2.3,2.3]$} \\
$\begin{array}{l}\text { Ternary } \\
{[3]}\end{array}$ & Nonstationary & $C^{1}$ & 4 & {$[-3,3]$} \\
$\begin{array}{l}\text { Ternary } \\
{[4]}\end{array}$ & Nonstationary & $C^{2}$ & 4 & {$[-3,3]$} \\
$\begin{array}{l}\text { Proposed } \\
(21)\end{array}$ & Nonstationary & $C^{2}$ & 3 & {$[-2.5,2.5]$} \\
\hline
\end{tabular}

Similarly, one can obtain

$$
\begin{aligned}
\sum_{j \in Z}^{\infty}\left|c_{4 j+1}^{(k)}-c_{4 j+1}\right|= & 4\left|a_{0}^{k}-\frac{7}{128}\right|+4\left|a_{2}^{k}-\frac{35}{128}\right| \\
& +8\left|a_{3}^{k}-\frac{5}{128}\right|+12\left|b_{0}^{k}-\frac{1}{16}\right|+4\left|b_{1}^{k}-\frac{9}{16}\right|, \\
\sum_{j \in Z}^{\infty}\left|c_{4 j+2}^{k)}-c_{4 j+2}\right|= & 8\left|a_{0}^{k}-\frac{7}{128}\right|+4\left|a_{1}^{k}-\frac{105}{128}\right| \\
& +4\left|a_{3}^{k}-\frac{5}{128}\right|+12\left|b_{0}^{k}-\frac{1}{16}\right|+4\left|b_{1}^{k}-\frac{9}{16}\right| .
\end{aligned}
$$

From Lemma 5, we have $\sum_{k=0}^{\infty} 4^{k}\left|a_{0}^{(k)}-7 / 128\right|<\infty$, $\sum_{k=0}^{\infty} 4^{k}\left|a_{1}^{(k)}-105 / 128\right|<\infty, \sum_{k=0}^{\infty} 4^{k}\left|a_{2}^{(k)}-35 / 128\right|<\infty$, $\sum_{k=0}^{\infty} 4^{k}\left|a_{3}^{(k)}-5 / 128\right|<\infty, \sum_{k=0}^{\infty} 4^{k}\left|b_{0}^{(k)}-1 / 16\right|<\infty$, and $\sum_{k=0}^{\infty} 4^{k}\left|b_{1}^{(k)}-9 / 16\right|<\infty$. So, it can be written as

$$
\sum_{k=0}^{\infty} 4^{k}\left\|S_{c^{(k)}}-S_{c}\right\|_{\infty}<\infty
$$

Thus, the scheme corresponding to symbol $c^{(k)}(z)$ is $C^{1}$ and hence the scheme (21) is $C^{2}$.

Figure 1 shows the reproduction of the unit circle and ellipse by the proposed scheme (21), taking regular hexagon as control points for circle.

In Figure 2, twelve points have been taken as control points on the parametric curves of asteroid: $x(t)=3 \cos t+$ $\cos 3 t, y(t)=3 \sin t-\sin 3 t$ and cardioid: $x(t)=(1-$ $\cos t) \sin t, y(t)=(1-\cos t) \cos t$ to reconstruct the asteroid and cardioid, respectively, by the proposed scheme.

Figure 3 shows the behavior of basic limit function for different values of $\alpha$. 


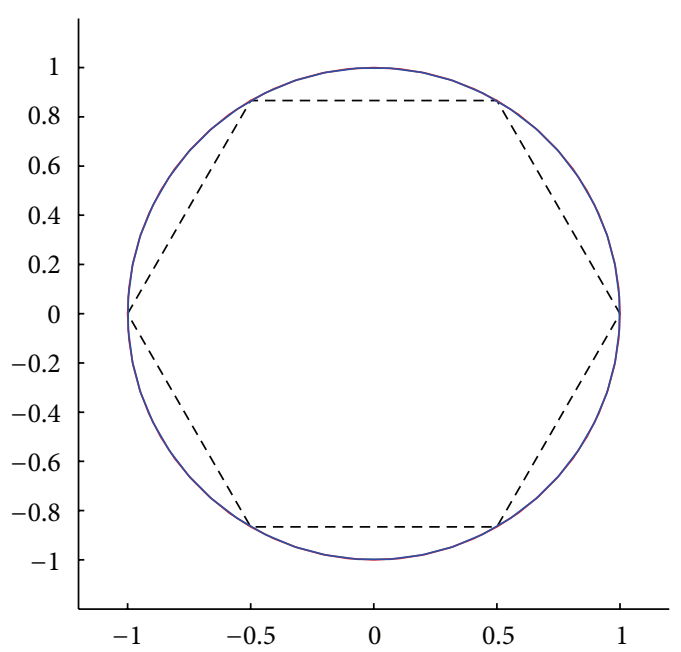

(a)

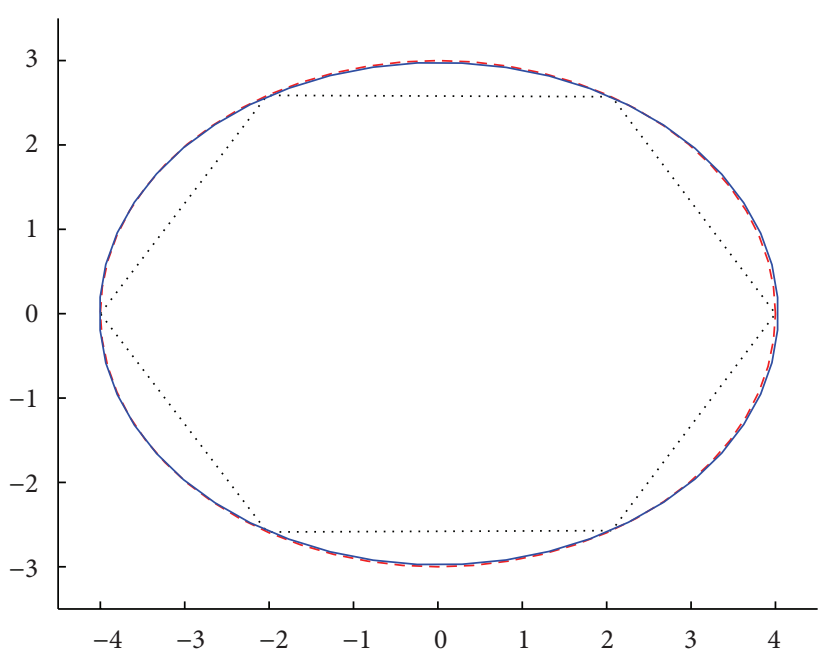

(b)

FIGURE 1: The reproduction of the unit circle and ellipse by the proposed scheme.

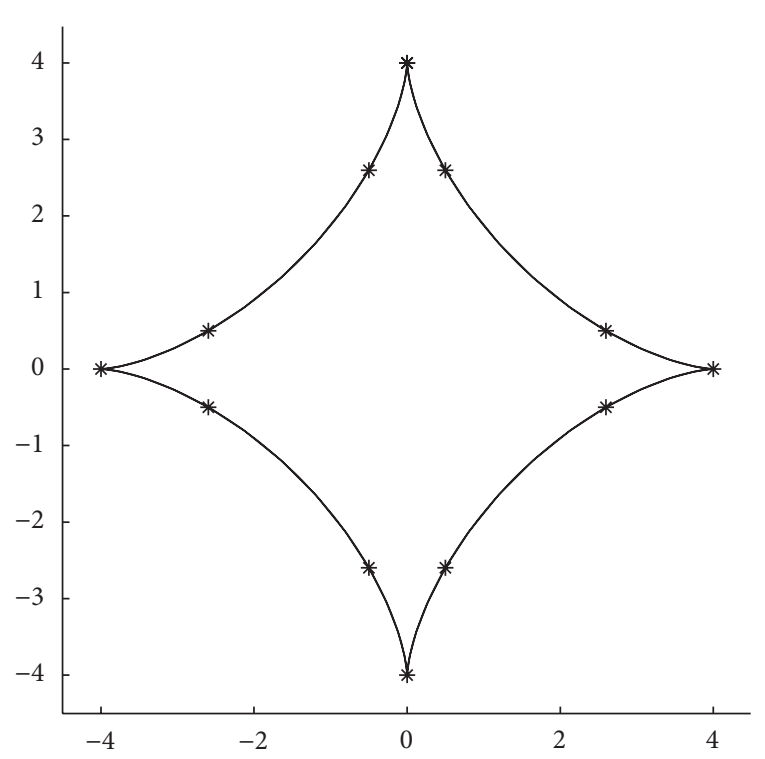

(a)

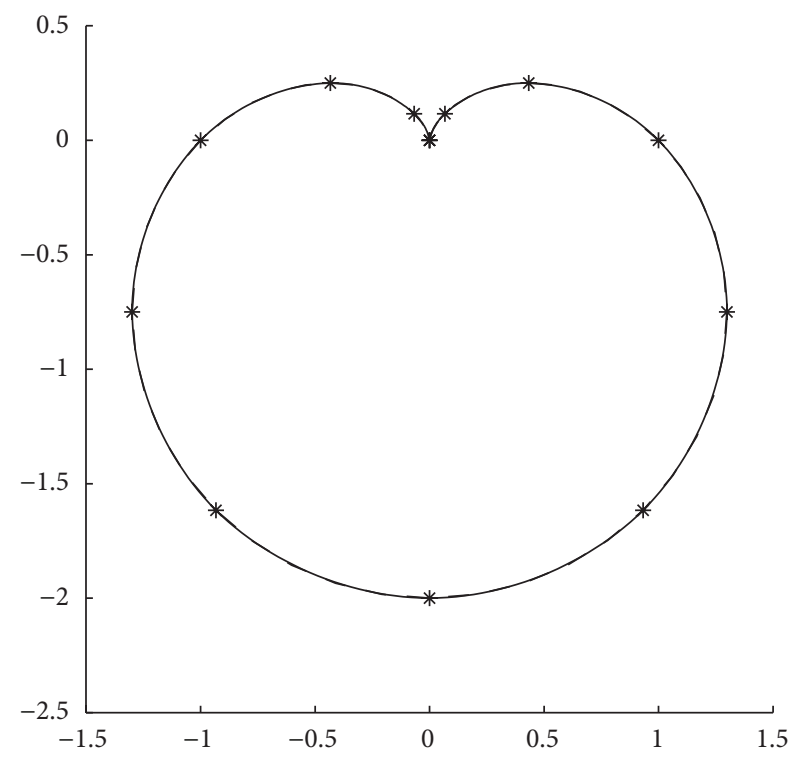

(b)

FIGURE 2: The reconstruction of asteroid and cardioid by the proposed scheme.

\section{Properties of the Scheme}

In this section some properties (approximation order, reproduction property, support size, and symmetry of basic limit function) are being discussed. The results show that the proposed scheme with the same approximation order has the higher smoothness and smaller support than the existing 4point interpolating subdivision schemes (see also Table 1).

Another advantage (see also [14]) of the scheme is the computational cost. In one subdivision step, for a sequence of $n$ points the proposed scheme requires $12 n$ multiplications and $9 n$ additions. On the other hand, computing the same number of $4 n$ refined points with the binary scheme requires two subdivision steps with a total cost of $18 n$ multiplications and $9 n$ additions, so the quaternary scheme is more efficient.
The basic limit function of the scheme is symmetric about $Y$-axis for the data

$$
f_{i}^{0}= \begin{cases}1, & i=0 \\ 0, & i \neq 0\end{cases}
$$

Theorem 9 (see [3]). The basic limit function $F$ is symmetric about the $Y$-axis.

Proof. Let $F$ denote the basic limit function and define $D_{n}:=$ $\left\{i / 4^{n} \mid i \in Z\right\}$ such that restriction of $F$ to $D_{n}$ satisfies $F\left(i / 4^{n}\right)=f_{i}^{n}$ for all $i \in Z$. The symmetry of basic function is proved using induction on $n$.

It can be observed that $F(i)=F(-i)$ for all $i \in Z$; thus $F\left(i / 4^{n}\right)=F\left(-i / 4^{n}\right)$ for all $i \in Z$ and $n=0$. Assume that 


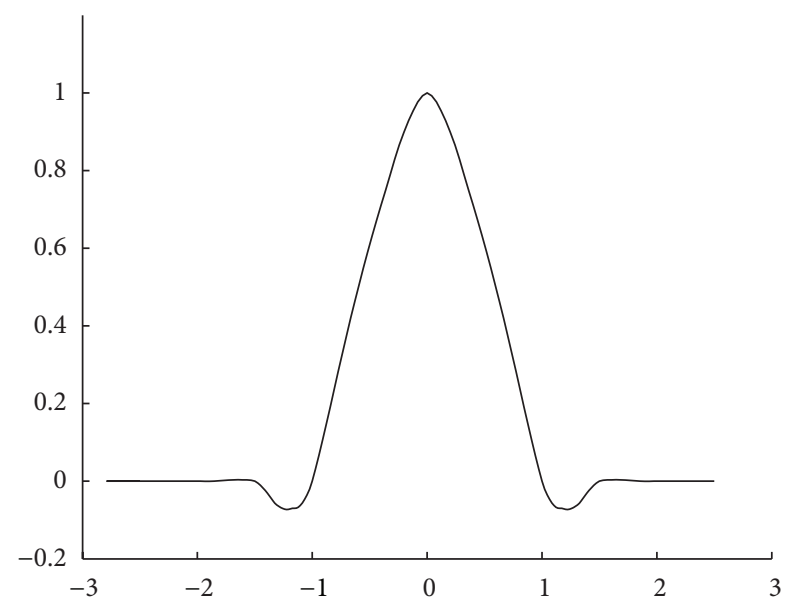

(a)

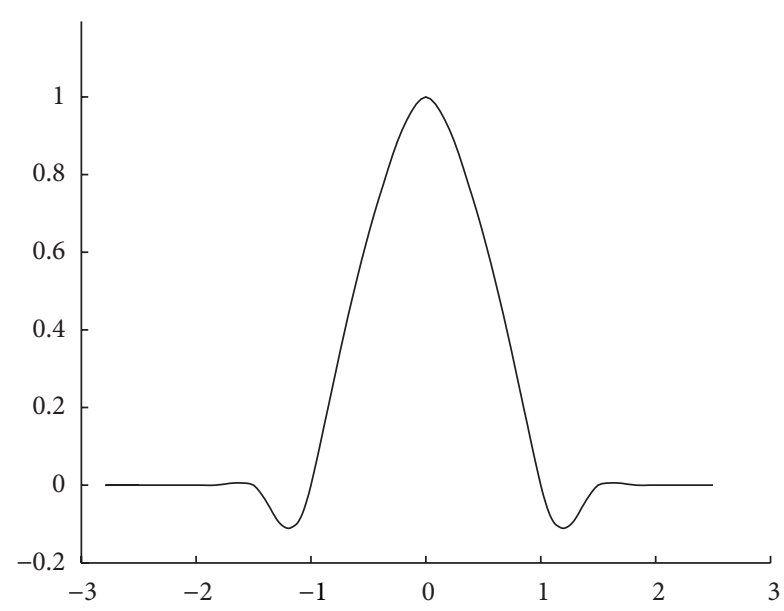

(b)

FIGURE 3: Basic limit function of proposed scheme for different values of $\alpha$.

$F\left(i / 4^{n}\right)=F\left(-i / 4^{n}\right)$ for all $i \in Z$ and then $f_{i}^{n}=f_{-i}^{n}$ for all $i \in Z$. It may be observed that

$$
\begin{aligned}
F\left(\frac{4 i}{4^{n+1}}\right) & =f_{4 i}^{n+1}=f_{-4 i}^{n+1}=F\left(-\frac{4 i}{4^{n+1}}\right), \\
F\left(\frac{4 i+1}{4^{n+1}}\right) & =f_{4 i+1}^{n+1} \\
& =-a_{0}^{n} f_{i-1}^{n}+a_{1}^{n} f_{i}^{n}+a_{2}^{n} f_{i+1}^{n}-a_{3}^{n} f_{i+2}^{n} \\
& =-a_{3}^{n} f_{-i-2}^{n}+a_{2}^{n} f_{-i-1}^{n}+a_{1}^{n} f_{-i}^{n}-a_{0}^{n} f_{-i+1}^{n} \\
& =f_{-4 i-1}^{n+1}=F\left(-\frac{4 i+1}{4^{n+1}}\right) .
\end{aligned}
$$

Similarly

$$
\begin{aligned}
& F\left(\frac{4 i+2}{4^{n+1}}\right)=f_{4 i+2}^{n+1}=f_{-4 i-2}^{n+1}=F\left(-\frac{4 i+2}{4^{n+1}}\right), \\
& F\left(\frac{4 i+3}{4^{n+1}}\right)=f_{4 i+3}^{n+1}=f_{-4 i-3}^{n+1}=F\left(-\frac{4 i+3}{4^{n+1}}\right) .
\end{aligned}
$$

Hence, $F\left(i / 4^{n}\right)=F\left(-i / 4^{n}\right)$ for all $i \in Z$ and $n=Z_{+}$; thus from the continuity of $F, F(x)=F(-x)$ holds for all $x \in R$, which completes the required result.

In the following the support of basic limit function of the proposed scheme has been calculated generated by the control point $f_{i}^{0}$

Theorem 10. The basic limit function $F$ defined by the scheme vanishes outside $[-7 / 3,7 / 3]$.

Proof. Define $t_{n}$ recursively by taking $t_{n+1}=t_{n}+1 / 4^{n+1}$ with $t_{0}=0$. It can be observed that $t_{n} \in D_{n}^{1}$ but $t_{n} \notin D_{n-1}^{1}$. We claim that restriction of $F$ to $D_{n}$ vanishes outside $\left[-t_{n}, t_{n}\right]$. Since $F$ is symmetric, it is enough to prove that $F$ vanishes outside $\left[0, t_{n}\right]$. Thus, after $k$ th subdivision step, the furthest nonzero vertex on the left/right will be at $(7 / 4) \sum_{j=0}^{k-1}\left(1 / 4^{j}\right)$. So, it can be concluded that the left or right influence of the original nonzero vertex will propagate along $\lim _{n \rightarrow \infty} t_{n}=$ $\sum_{j=0}^{\infty}(7 / 4)(1 / 4)^{j}=(7 / 4)(1 /(1-(1 / 4)))=7 / 3$. It can be concluded that $\left[-t_{n}, t_{n}\right]$ is contained in $[-7 / 3,7 / 3]$ for all $n$ and $\lim _{n \rightarrow \infty}\left[-t_{n}, t_{n}\right]=[-7 / 3,7 / 3]$, which is the required result.

Theorem 11 (see [15]). A convergent subdivision scheme that reproduces polynomial $\pi_{n}$ has an approximation order $n+1$.

So in the light of the previous theorem, the proposed scheme has approximation order 4 because the construction of mask is formulated through the cubic trigonometric polynomials.

The polynomial reproduction property has its own importance, as the reproduction property of the polynomials up to certain degree $d$ implies that the scheme has $d+1$ approximation order. For this, polynomial reproduction can be made from initial data which has been sampled from some polynomial functions. So, in view of the previous theorem, scheme (32) has approximation order 4.

\section{Conclusion}

A 4-point novel quaternary interpolating nonstationary subdivision scheme has been developed which generates the family of $C^{2}$ limiting curves and its limiting function has support on $[-7 / 3,7 / 3]$. The nonstationary scheme is associated with trigonometric polynomials which reproduces the functions of linear spaces spanned by $\{1, \sin (\alpha x), \cos (\alpha x)\}$ for $0<\alpha<$ $\pi / 3$. The comparison shows that, with existing stationary and nonstationary interpolating schemes, the proposed scheme is better. Moreover, it also can reconstruct or reproduce the conic sections, asteroids, and cardioids.

\section{References}

[1] C. Beccari, G. Casciola, and L. Romani, "A non-stationary uniform tension controlled interpolating 4-point scheme reproducing conics," Computer Aided Geometric Design, vol. 24, no. 1, pp. 1-9, 2007. 
[2] M. K. Jena, P. Shunmugaraj, and P. C. Das, "A non-stationary subdivision scheme for curve interpolation," ANZIAM Journal, vol. 44, pp. E216-E235, 2003.

[3] C. Beccari, G. Casciola, and L. Romani, "An interpolating 4-point $C^{2}$ ternary non-stationary subdivision scheme with tension control," Computer Aided Geometric Design, vol. 24, no. 4, pp. 210-219, 2007.

[4] S. Daniel and P. Shunmugaraj, "Some interpolating nonstationary subdivision schemes," in Proceedings of International Symposium on Computer Science and Society, pp. 400-403, 2011.

[5] C. Conti and L. Romani, "Affine combination of B-spline subdivision masks and its non-stationary counterparts," BIT Numerical Mathematics, vol. 50, no. 2, pp. 269-299, 2010.

[6] S. S. Siddiqi and M. Younis, "Ternary three point non-stationary subdivision scheme," esearch Journal of Applied Sciences, Engineering and Technology, vol. 4, no. 13, pp. 1875-1882, 2012.

[7] S. S. Siddiqi and M. Younis, "Construction of $m$-point binary approximatin subdivision schemes," Applied Mathematics Letters, vol. 26, no. 3, pp. 337-343, 2013.

[8] N. Dyn, D. Levin, and J. A. Gregory, "A 4-point interpolatory subdivision scheme for curve design," Computer Aided Geometric Design, vol. 4, no. 4, pp. 257-268, 1987.

[9] M. F. Hassan, I. P. Ivrissimitzis, N. A. Dodgson, and M. A. Sabin, "An interpolating 4-point $C^{2}$ ternary stationary subdivision scheme," Computer Aided Geometric Design, vol. 19, no. 1, pp. $1-18,2002$.

[10] N. Dyn, "Tutorials on multresolution in geometric modelling," in Summer Schools Lectures Notes Series: Mathematics and Visualization, A. Iske, E. Quak, and M. S. Floater, Eds., Springer, 1995.

[11] N. Dyn and D. Levin, "Analysis of asymptotically equivalent binary subdivision schemes," Journal of Mathematical Analysis and Applications, vol. 193, no. 2, pp. 594-621, 1995.

[12] T. Lyche, "A newton form for trigonometric Hermite interpolation," BIT Numerical Mathematics, vol. 19, no. 2, pp. 229-235, 1979.

[13] N. Dyn and D. Levin, "Subdivision schemes in geometric modeling," Acta Numerica, vol. 11, pp. 73-144, 2002.

[14] C. Conti and K. Hormann, "Polynomial reproduction for univariate subdivision schemes of any arity," Journal of Approximation Theory, vol. 163, no. 4, pp. 413-437, 2011.

[15] N. Dyn, "Subdivision schemes in computer aided geometric design," in Advances in Numerical Analysis, Subdivision Algorithms and Radial Functions, W. A. Light, Ed., pp. 36-104, Oxford University Press, 1992. 

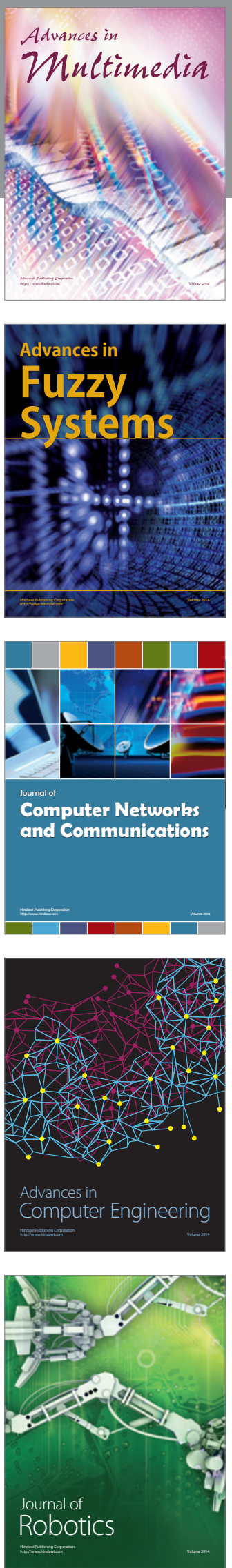

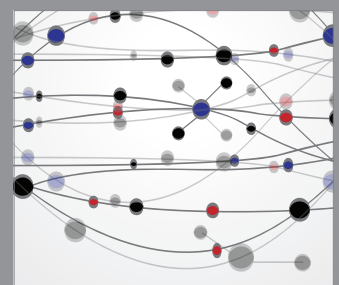

The Scientific World Journal
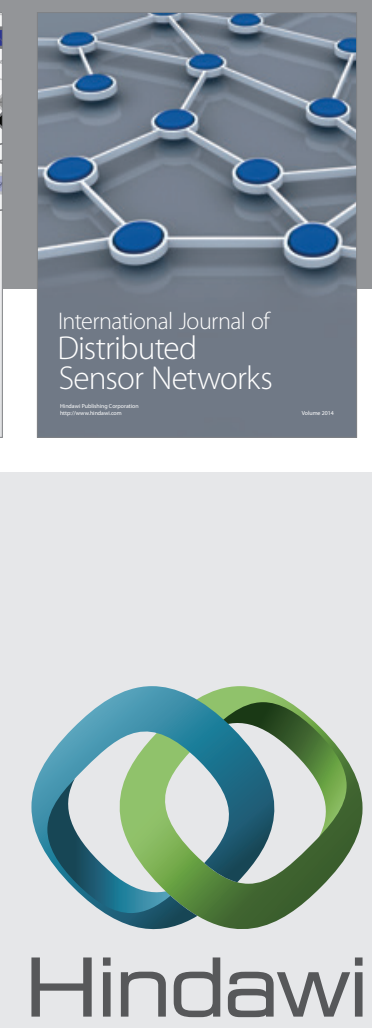

Submit your manuscripts at

http://www.hindawi.com
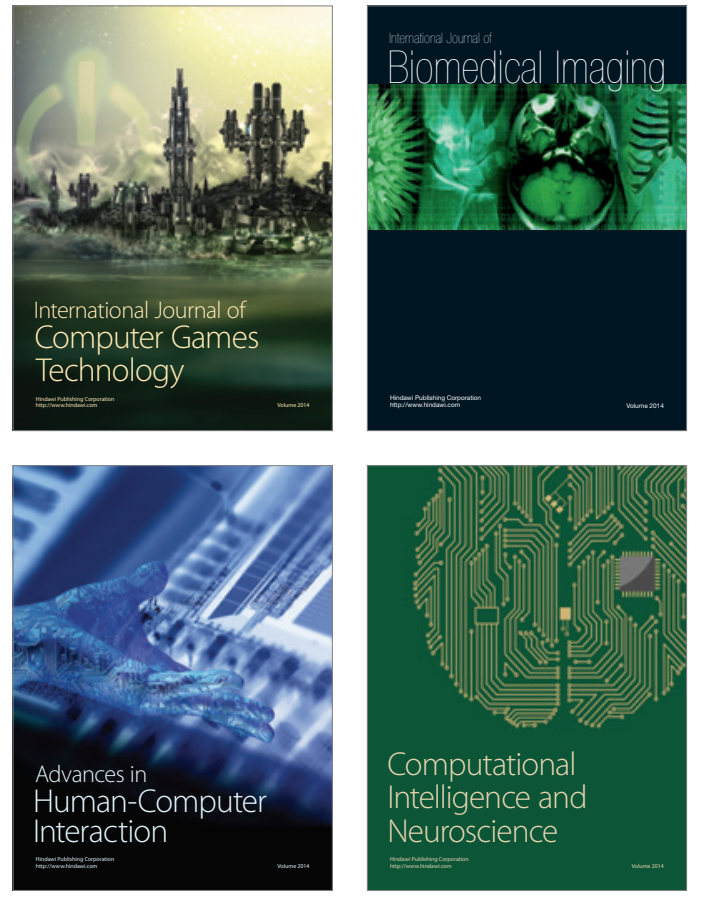
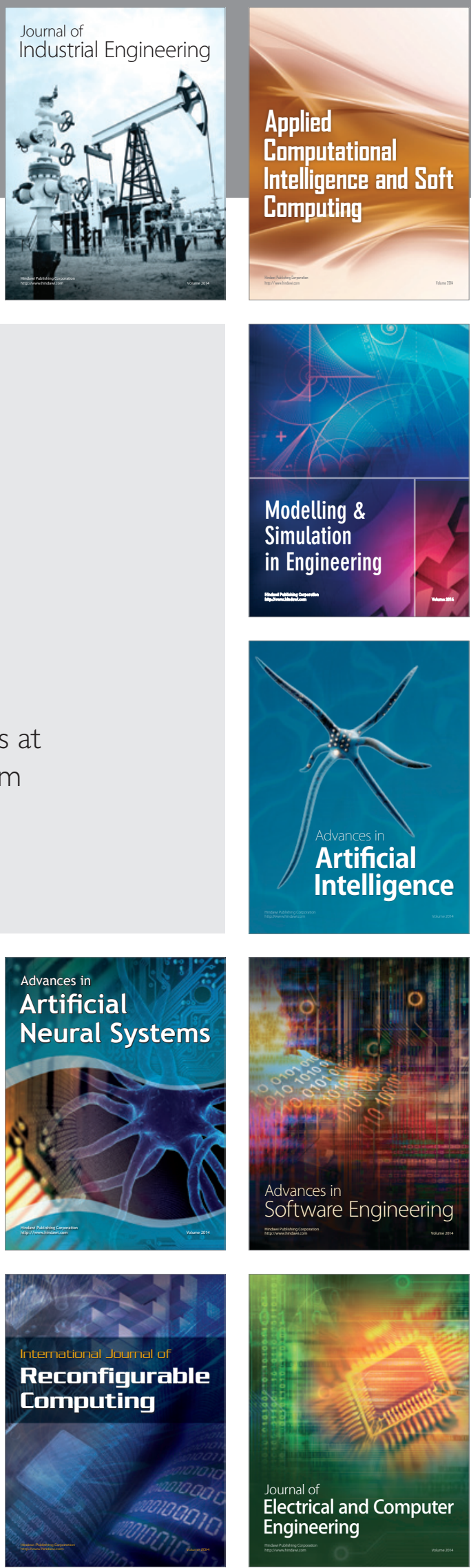